\title{
Carrier Screening
}

National Human Genome Research Institute (NHGRI)

\section{Source}

National Human Genome Research Institute (NHGRI). Carrier Screening.

Carrier screening is a type of genetic testing performed on people who display no symptoms for a genetic disorder but may be at risk for passing it on to their children. A carrier for a genetic disorder has inherited one normal and one abnormal allele for a gene associated with the disorder. A child must inherit two abnormal alleles in order for symptoms to appear. Prospective parents with a family history of a genetic disorders are candidates for carrier screening. 